\title{
The effect of induced anxiety on cognition: threat of shock enhances aversive processing in healthy individuals
}

\author{
Oliver J. Robinson - Allison M. Letkiewicz • \\ Cassie Overstreet • Monique Ernst • Christian Grillon
}

Published online: 12 April 2011

(C) Psychonomic Society, Inc. (outside the USA) 2011

\begin{abstract}
Individuals with anxiety disorders demonstrate altered cognitive performance including (1) cognitive biases towards negative stimuli (affective biases) and (2) increased cognitive rigidity (e.g., impaired conflict adaptation) on affective Stroop tasks. Threat of electric shock is frequently used to induce anxiety in healthy individuals, but the extent to which this manipulation mimics the cognitive impairment seen in anxiety disorders is unclear. In this study, 31 healthy individuals completed an affective Stroop task under safe and threat-of-shock conditions. We showed that threat (1) enhanced aversive processing and abolished a positive affective bias but (2) had no effect on conflict adaptation. Threat of shock thus partially models the effects of anxiety disorders on affective Stroop tasks. We suggest that the affective state of anxiety - which is common to both threat and anxiety disorders - modulates the neural inhibition of subcortical aversive processing, whilst pathologies unique to anxiety disorders modulate conflict adaptation.
\end{abstract}

Keywords Anxiety - Threat of shock - Affective bias . Conflict adaptation $\cdot$ Resilience $\cdot$ BIS/BAS

Anxiety disorders are alarmingly prevalent-over $25 \%$ of the population is likely to suffer from one of these disorders at some point in their lives (Kessler, Berglund, Demler, Jin, Merikangas, \& Walters, 2005) - and they carry enormous economic and emotional costs for both individuals and society (Beddington et al., 2008). In addition to their

O. J. Robinson $(\square) \cdot$ A. M. Letkiewicz $\cdot$ C. Overstreet $\cdot$

M. Ernst $\cdot$ C. Grillon

Mood and Anxiety Disorders Program, NIMH,

15K North Drive, MSC 2670,

Bethesda, MD 20892, USA

e-mail: oliver.j.robinson@gmail.com affective symptoms, anxiety disorders are associated with alterations in cognitive performance. Individuals with anxiety disorders, for example, demonstrate affective biases towards aversive stimuli, an effect that can serve to both stimulate and uphold a state of anxiety (Mogg \& Bradley, 2005). One task that can reveal these biases is the affective Stroop task (Becker, Rinck, Margraf, \& Roth, 2001; Etkin, Prater, Hoeft, Menon, \& Schatzberg, 2010), in which subjects are asked to identify stimuli in the face of (congruent or incongruent) distractors. Anxiety disorders are associated with negative affective biases (i.e., bias towards processing aversive stimuli and away from appetitive stimuli) on classic versions of the affective Stroop (where affective distractors compete for attention with nonaffective targets; Becker et al., 2001). Moreover, on an adaptation of the Stroop task in which the targets and distractors are both emotional stimuli (which is typically more effective at eliciting response conflict effects in healthy adults, because both targets and distractors compete for affective responses; Buhle, Wager, \& Smith, 2010; Etkin, Egner, Peraza, Kandel, \& Hirsch, 2006), anxiety disorders have also been shown to abolish conflict adaptation. More specifically, although healthy individuals are slower to respond to incongruent than to congruent stimuli (due to interference from the incongruent distractor), the response to incongruent (or conflict) trials is faster when the previous trial was also incongruent. This is referred to as conflict adaptation and is an example of cognitive flexibility. Flexibility on this adapted Stroop task (Etkin et al., 2010) is absent in individuals with generalized anxiety disorder and panic disorder (Chechko, Wehrle, Erhardt, Holsboer, Czisch, \& Sämann, 2009; Etkin et al., 2010), suggesting that anxiety disorders are associated with reduced cognitive flexibility in addition to the negative affective biases. 
Affective biases and altered cognitive flexibility may, however, be driven by distinct mechanisms. Conflict adaptation may depend on "top-down," cortically mediated cognitive control (Egner \& Hirsch, 2005; Etkin et al., 2006), whilst affective biases may result from a combination of altered "bottom-up," subcortical affective processing (Robinson \& Sahakian, 2009b) and "top-down" control of these processes (Bishop, 2007; Shackman, Maxwell, McMenamin, Greischar, \& Davidson, 2011). Any of these processes, alone or in combination, may be impacted by anxiety disorders. As such, understanding the normative underlying mechanisms of these emotional and cognitive processes is critical for a full comprehension of their dysfunction in psychopathology (Kupfer, First, \& Regier, 2002).

One approach to clarifying the role of a given symptom in the overall pathology of a psychiatric disorder is to reproduce that symptom in healthy individuals (or animal models) and to examine its effect on task performance. For anxiety disorders, this can be achieved using various procedures, including threat of shock (Grillon, 2008a, 2008b), fear conditioning (Bishop, 2007), and acute tryptophan depletion (Robinson \& Sahakian, 2009a, 2009b). Here, we focus on the threat-ofshock paradigm, in which healthy individuals receive infrequent and unpredictable electric shocks. During threat periods, subjects demonstrate robust increases in subjective and physiological signs of anxiety (Grillon, 2008b), increased vigilance towards novel stimuli (Cornwell et al., 2007), elevated amygdala reactivity (Phelps et al., 2001), attenuated reward anticipation (Ryan \& Diego, 2006), and impaired spatial working memory (Erk, Kleczar, \& Walter, 2007; Lavric, Rippon, \& Gray, 2003; Shackman et al., 2006). A large number of studies have demonstrated elevated startle reflex under threat of shock (Grillon, 2008b), but the extent to which experimental anxiety induced by threat of shock mimics other cognitive symptoms of anxiety, such as affective biases and cognitive flexibility, is surprisingly understudied. Given the effects of clinical anxiety on cognitive performance, threat of shock in healthy individuals should enhance aversive processing and reduce cognitive flexibility, as indexed by conflict adaptation. However, to the best of our knowledge, no studies have directly assessed these effects. Here we therefore examine whether the effects of threat of shock in healthy individuals mimic the affective bias and reduced cognitive flexibility seen in individuals with anxiety disorders on the affective Stroop task (Egner, Etkin, Gale, \& Hirsch, 2008; Etkin et al., 2006). Much can be gained by developing experimental models of anxiety, but not all facets of anxiety can be modelled in a single paradigm, and it is critical to delineate the boundaries of our experimental models.

Manipulating anxiety using the threat-of-shock paradigm has significant advantages over measuring anxiety using state and trait anxiety questionnaires (Hettema, Neale, \& Kendler,
2001; Krug \& Carter, 2010; Miu, Heilman, \& Houser, 2008; Osinsky, Alexander, Gebhardt, \& Hennig, 2010; Shackman et al., 2006). First, anxiety level is actually manipulated within subjects, and thus findings can be linked directly to this controlled, experimental manipulation. Second, in questionnaire-based studies the effect of trait anxiety could be due to other facets of personality rather than to anxiety per se, which could obscure the interpretation of findings. Finally, the threat paradigm still allows for the examination of the effects of individual differences in trait anxiety, enabling us to measure the impact of individual differences in BIS/BAS (behavioural inhibition system/behavioural activation system) processing in the present study. The BIS/ BAS scale is a widely used measure sensitive to individual differences in appetitive approach (BAS) and aversive avoidance (BIS) (Carver \& White, 1994; Simon et al., 2009). The BIS scale has been shown to correlate with anxiety disorders, with higher scores indicating higher levels of anxiety (Muris, Merckelbach, Schmidt, Gadet, \& Bogie, 2001; for a review, see McNaughton \& Gray, 2000). High BIS scores are also associated with a number of anxietyrelated traits, including increased amygdala reactivity to fearful faces (Cools et al., 2005), increased amygdala grey matter (Barrós-Loscertales et al., 2006), impaired spatial working memory (Shackman et al., 2006), elevated no-go $\mathrm{N} 2$ and event-related negativity (ERN) amplitudes (Amodio, Master, Yee, \& Taylor, 2008), increased impact of daily stressors on negative affect (Gable, Reis, \& Elliot, 2000), and reduced striatal activity to reward (Simon et al., 2009). As such, amongst psychiatrically healthy individuals, higher scorers on the BIS may indicate risk for the cognitive disruptions seen in affective disorders (Shackman et al., 2006), although it should be noted that recent studies have failed to find an effect of BIS/BAS on conflict adaptation (Osinsky et al., 2010). In a secondary analysis, we therefore assessed the impact of individual differences in BIS and BAS reward sensitivity on threat-modulated cognitive performance.

In sum, we tested whether threat of shock successfully mimics the cognitive disruption seen in anxiety disorders. We predicted that, consistent with the effects of anxiety disorders on cognitive performance, threat of shock, relative to safety, would (1) facilitate negative affective processing and (2) disrupt conflict adaptation on the affective Stroop task.

\section{Method and materials}

\section{Subjects}

A total of 31 physically and mentally healthy volunteers ( $28 \pm$ 7 years; 16 women) signed written informed consent approved by the NIMH Human Investigation Review Board. 
Subjects responded to advertisements asking for healthy volunteers. Physical and mental health of the participants was determined by a physical examination performed by a physician, a clinical interview conducted by a trained psychologist using the Structured Clinical Interview for the DSM-IV (SCID; First, Spitzer, Gibbon, \& Williams, 2002), and self-report of medication and drug use confirmed by urine toxicology analysis. All participants were required to be free of a medical condition that could interfere with the study, past or current psychiatric disorders, and current use of drugs or psychoactive medications. Other individual clinical characteristics were assessed using the BIS/BAS (Carver \& White, 1994), the Beck Depression Inventory (Beck, Ward, Mendelson, Mock, \& Erbaugh, 1961), the Spielberger State-Trait Anxiety Inventory (Spielberger, 1983), and the fear-of-pain scales (Osman, Breitenstein, Barrios, Gutierrez, \& Kopper, 2002); see Table 1.

\section{Procedures}

Subjects completed the Stroop task followed by a posttask face-rating task. The Stroop task was performed under safe and threat conditions. The threat procedure was based on previous paradigms (e.g., Cornwell et al., 2007; Hasler, van der Veen, Grillon, Drevets, \& Shen, 2010). Prior to starting the Stroop task, subjects were told that they would receive between two and five shocks, and they went through a shock workup procedure to control for individual differences in the level of shock experienced. The shock level was gradually increased until subjects rated the shock as moderately painful. No more

Table 1 Demographic characteristics

\begin{tabular}{lllll}
\hline Demographic Measure (Total $N=31$ ) & Score & SEM \\
\hline Gender & 16 female & & \\
Ethnicity & 17 Caucasian & & \\
Age & 28.0 & 7.6 & 3.2 & \\
Beck Depression Inventory & 2.2 & 3.9 & \\
Behavioral Inhibition Scale (BIS) & 18.6 & 2.6 & 2.0 \\
subgroups & 15.8 & 22.0 & 2.0 & \\
Behavioral Activation Scale (BAS) & 17.8 & & & \\
$\quad$ Reward Responsiveness & & & 1.8 & 0.5 \\
subgroups & 16.4 & 19.4 & 2.4 & \\
Behavioral Activation Scale (BAS) & 12.2 & & & \\
$\quad$ Fun Seeking & & & 2.3 & \\
Behavioral Activation Scale (BAS) & 11.4 & & & \\
$\quad$ Drive & 67.2 & & 19.0 & \\
Fear-of-Pain Scale & 29.7 & 7.1 & \\
Spielberger (1983) Trait Anxiety & 26.5 & 5.5 & \\
Spielberger (1983) State Anxiety & &
\end{tabular}

$S E M=$ standard error of the mean. than four shocks were administered during this workup. To minimize habituation to the shock and maximize unpredictability, participants actually received two shocks during the experiment, each of which came at the end of threat blocks. Trials immediately following shock were excluded from analysis. Shock was applied to the wrist using a commercial system (Psylab, London, U.K.). Shock electrodes remained in place for the entirety of the experiment.

The effectiveness of the threat condition was assessed via $10-\mathrm{cm}$ visual analogue scales (VAS) asking subjects to rate how "anxious," "afraid," and "happy" they felt following each safe and threat condition.

Stroop task The task was based on that used by both Egner and Etkin (Egner et al., 2008; Etkin et al., 2006; see Fig. 1). A face stimulus (happy/fearful) overlaid with a word ("happy"/"fear") appeared on a white background. The stimuli were either congruent (e.g., fearful face + "fear"), or incongruent (e.g., happy face + "fear") Ekman faces (Ekman \& Friesen, 1976). The subject was asked to identify the emotion of the face whilst ignoring the emotion of the word (N.B.: This differs from more traditional emotional Stroop tasks, in which subjects are asked to identify nonemotional aspects [e.g., colour] of emotional stimuli [e.g., words]; Ernst, 2010). There were five male and five female faces, each of which displayed either happy or fearful emotion (20 images total). Consistent with the original task (Egner et al., 2008; Etkin et al., 2006), stimuli were presented for $1,000 \mathrm{~ms}$, followed by a jitter of 3,000 $5,000 \mathrm{~ms}$ (mean 4,000 ms), during which a central fixation cross was displayed on a white background. Stimuli were presented in pseudorandom order (counterbalanced for equal numbers of congruent-congruent $[\mathrm{CC}]$, congruentincongruent $[\mathrm{CI}]$, incongruent-congruent $[\mathrm{IC}]$, and incongruent-incongruent [II] stimulus sequences). The sex and facial expression of the target stimuli were counterbalanced across these trial types, and the proportion of response repetitions to response alternations was the same (50\%) for all trial sequence types. There were no direct repetitions of the same face, to avoid a priming effect.

The task was organised into (eight total) alternating threat (four) and safe (four) conditions (order counterbalanced) that were signalled to the subjects by the text "You may receive an electric shock at some point during the next set of trials" and "You are now safe from shock for this next set of trials." Subjects were informed that they would receive shocks in the threat, but not in the safe, conditions (order counterbalanced). Each condition consisted of 48 trials (50 during shock blocks) lasting approximately $3.1 \mathrm{~min}$; the duration of the whole task was 384 trials, or approximately 25 min total. Subjects completed an additional 16 practice trials prior to commencing the experiment. 


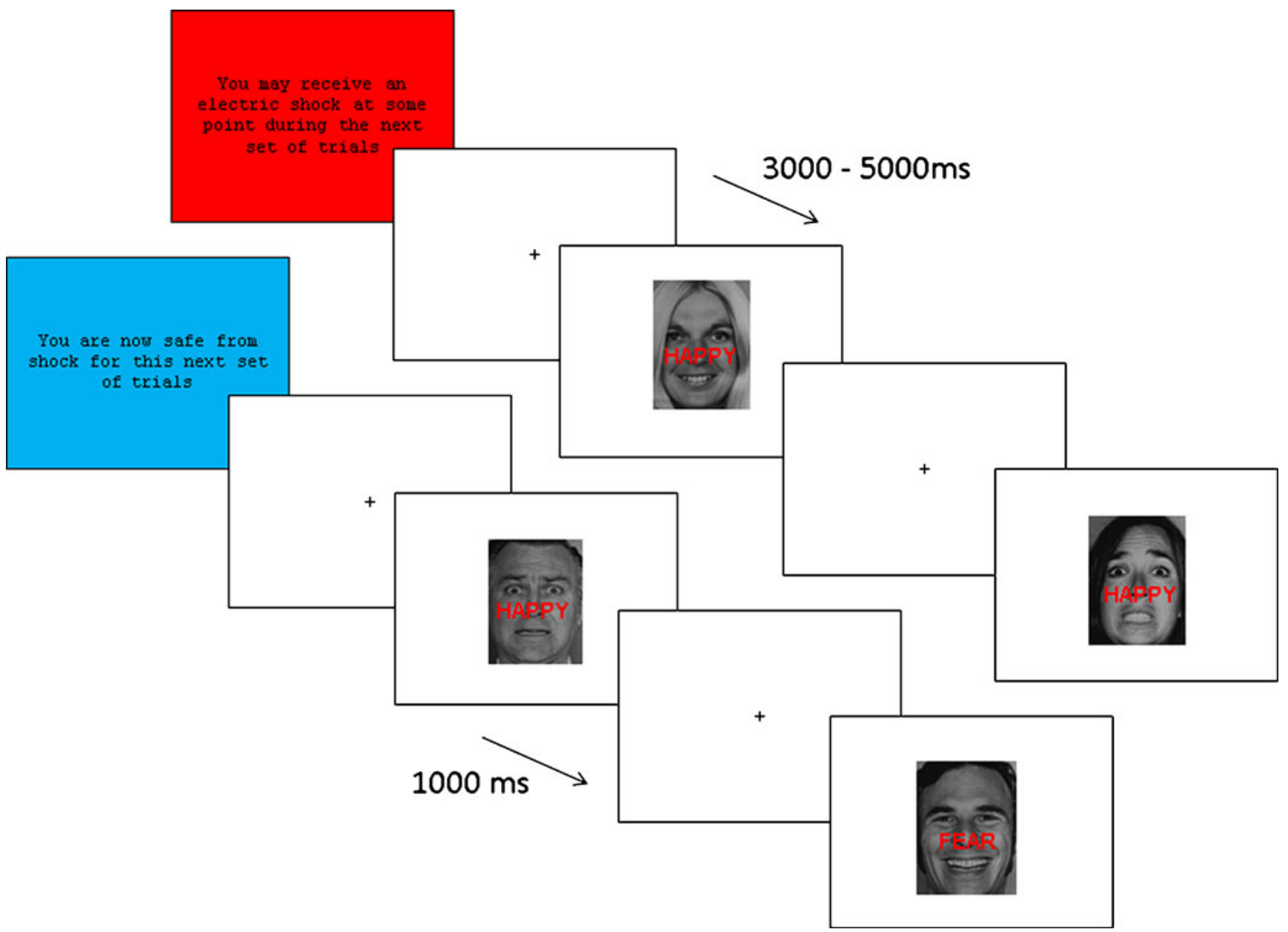

Fig. 1 The task was based on that used by both Egner and Etkin (Egner et al., 2008; Etkin et al., 2006). The subject was asked to identify the emotion of the face whilst ignoring the emotion of the

Analytic methods Performance was assessed for accuracy and reaction time (RT). Since each trial was defined as a function of the preceding trial, trials on which the preceding trials were errors were excluded. Additionally, to ensure consistency with Egner et al. (2008), RT was calculated on correct responses only. RT and accuracy were assessed in separate general linear models, with condition (threat/safe), previous trial (incongruent/congruent), current trial (incongruent/congruent), and target valence (fearful/happy) as within-subjects factors.

In order to examine the effect of individual differences in behavioural avoidance and reward seeking sensitivity on cognitive processing and threat response, subjects were divided according to median split scores on the BIS and BAS reward-seeking scales (Carver \& White, 1994). These median split BIS (high vs. low) and BAS (high vs. low) groups were individually included in secondary ANOVAs as between-subjects factors.

Conflict adaptation was assessed by examining the interaction between the congruency of the previous and word. The task was organised into (eight total) alternating threat (four) and safe (four) conditions, each containing 48 trials (plus 2 extra trials following the shock)

current trials. Affective biases (differences in response to fearful vs. happy faces) were assessed by examining performance on current target valence. Interactions were further assessed with post-hoc ANOVAs (Bonferroni corrected) and correlation according to Spearman's rho.

Face rating post-task To assess the comparability of the different face stimuli across valences, a posttask was performed in which subjects were presented with each of the 20 images used in the main experiment and asked to state the emotion on the face and then to rate "how much" the face expressed that emotion on a 1-5 Likert scale.

\section{Results}

RTs and error rates for all trial types are presented in Table 2. 
Table 2 Reaction times (in milliseconds) and error rates for each variable (as a function of trial type [II, IC, CI, CC] and valence [happy, fear])

$\mathrm{RT}=$ reaction time, $S E M=$ standard error of the mean, $\mathrm{H}=$ happy, $\mathrm{F}=$ fear, $\mathrm{II}=$ incongruent preceded by incongruent, $\mathrm{IC}=$ congruent preceded by incongruent, $\mathrm{CI}=$ incongruent preceded by congruent, $\mathrm{CC}=$ congruent preceded by congruent.

\begin{tabular}{|c|c|c|c|c|c|c|c|c|}
\hline & \multicolumn{2}{|l|}{ II } & \multicolumn{2}{|l|}{ IC } & \multicolumn{2}{|l|}{ CI } & \multicolumn{2}{|l|}{$\mathrm{CC}$} \\
\hline & $\mathrm{H}$ & F & $\mathrm{H}$ & $\mathrm{F}$ & $\mathrm{H}$ & $\mathrm{F}$ & $\mathrm{H}$ & $\mathrm{F}$ \\
\hline & \multicolumn{8}{|c|}{ Threat } \\
\hline Mean Error Rate & .20 & .19 & .13 & .10 & .22 & .17 & .12 & .13 \\
\hline SEM, Error Rate & .03 & .03 & .03 & .02 & .03 & .02 & .02 & .03 \\
\hline Mean RT & 738 & 740 & 706 & 705 & 755 & 738 & 703 & 716 \\
\hline \multirow[t]{2}{*}{$S E M, \mathrm{RT}$} & 10 & 11 & 11 & 10 & 12 & 11 & 11 & 12 \\
\hline & \multicolumn{8}{|c|}{ Safe } \\
\hline Mean Error Rate & .19 & .23 & .13 & .12 & .21 & .22 & .13 & .20 \\
\hline SEM, Error Rate & .02 & .03 & .02 & .02 & .03 & .03 & .03 & .03 \\
\hline Mean RT & 737 & 745 & 722 & 722 & 756 & 744 & 696 & 725 \\
\hline SEM, RT & 10 & 11 & 11 & 12 & 11 & 10 & 8 & 12 \\
\hline
\end{tabular}

\section{Manipulation check}

Subjects rated themselves as significantly more "anxious" $[F(1,29)=15, p=.001]$ and "afraid" $[F(1,29)=8$, $p=.007]$, but not less "happy" $[F(1,29)=2.8, p=.10]$, during the threat condition relative to the safe condition.

\section{Stroop task}

We used two separate general linear models to examine RT and accuracy, respectively.

\section{Reaction time}

The subjects were significantly faster on congruent $(712 \mathrm{~ms})$ than on incongruent $(745 \mathrm{~ms})$ trials [main effect of current trial: $F(1,30)=99, p<.001]$. Moreover, consistent with Egner et al. (2008), the RT analysis revealed a significant interaction between the congruency of the previous and current trials [previous $\mathrm{x}$ current trial interaction: $F(1,30)=5.9, p=.02]$, which was driven by significantly increased speed on II trials $(740 \mathrm{~ms})$ relative to CI trials (749 ms) [main effect of previous trial on incongruent trials: $F(1,30)=5.3, p=.03$ ] - that is, conflict adaptation. In contrast, RTs on congruent trials were not modulated by the congruency of the preceding trial [no effect of previous trial on congruent trials: $F(1,30)=1.2$, $p=.28$; Fig. 2a]. However, unlike Etkin et al. (2010), who found a significant difference between healthy and generalized-anxiety-disorder patients in the RT adjustment associated with conflict adaptation during incongruent trials, we found no effect of threat on conflict-related RT adjustment during incongruent trials [no condition $\mathrm{x}$ previous trial interaction in incongruent trials: $F(1,30)=$ 0.1, n.s.]. There was also no affective bias effect on RTs [no threat $\mathrm{x}$ valence interaction: $F(1,27)=1.5, p=.24]$, and as with previous research using Spielberger's state and trait anxiety scales (Osinsky et al., 2010), individual differences in BIS and BAS reward seeking did not impact conflict adaptation.

\section{Accuracy data}

Subjects were more accurate on congruent (.14 errors) than on incongruent trials (.21 errors) [main effect of current trial: $F(1,30)=55, p<.001]$. There was also an effect of threat of shock on affective bias [condition $\mathrm{x}$ valence interaction: $F(1,30)=8.6, p=.006]$. This interaction reflected a significantly enhanced accuracy on fearful faces under threat (.16 errors) relative to safe trials (.19 errors) [main effect of condition on fear face trials; $F(1,30)=13.8, p<.001]$ but no difference in response to happy trials across conditions [no effect of condition on happy trials; $F(1,30)=0.3$, n.s.]. Moreover, increased accuracy on happy (.16 errors) relative to fearful (.19 errors) trials in the safe condition led to a positive bias [main effect of valence under safe condition: $F(1,30)=4.6, p=.04$ ], which was not present in the threat condition [no effect of valence under threat: $F(1$, $30)=1.6, p=.22$; see Fig. 2b]. There was no conflict adaptation effect on accuracy [no previous $\mathrm{x}$ current trial interaction: $F(1,27)=1.9, p=.18]$.

Accuracy interaction with BIS When BIS was included as an extra factor in the accuracy ANOVA, there was a significant interaction between the high- and low-BIS groups in their accuracy on happy and fear face trials [valence $\mathrm{x}$ BIS interaction: $F(1,29)=11.8, p=.002$ ]. This interaction was driven by a significant positive affective bias [higher accuracy on happy (.15 errors) than on fearful (.19 errors) faces] in the low-BIS group [main effect of valence in low BIS: $F(1,29)=9.4, p=.005]$, which was not present in the high-anxiety group [no effect of valence in high BIS: $F(1,29)=3.3, p=.08$; see Fig. 3]. 

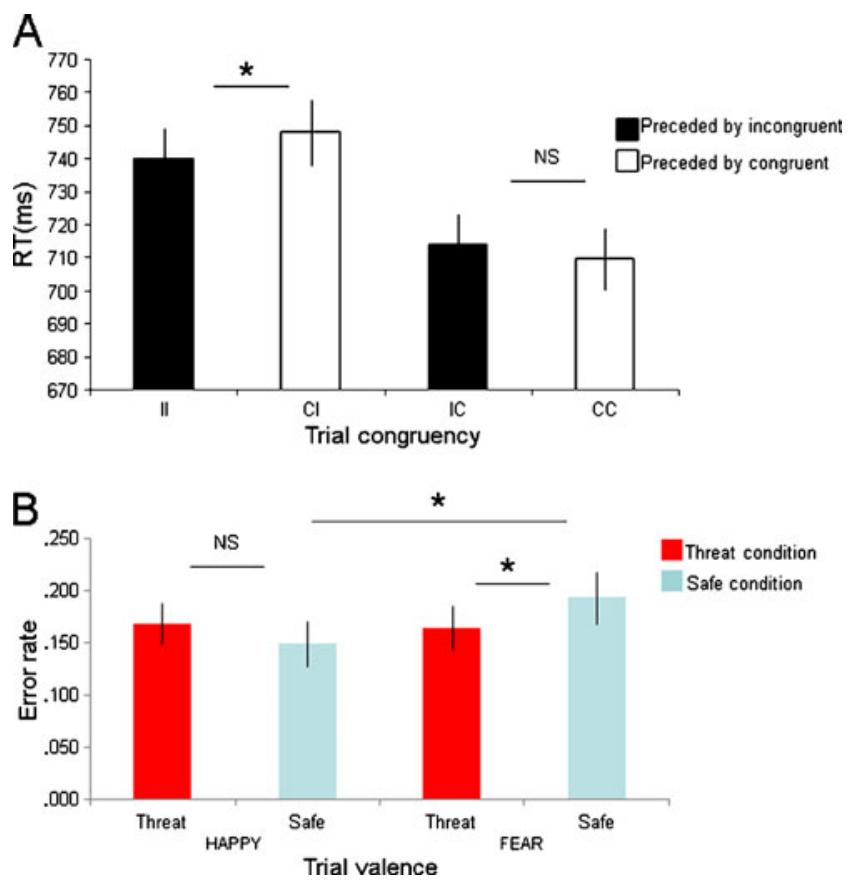

Fig. 2 Healthy individuals demonstrated conflict adaptation under both safe and threat conditions. Enhanced RTs are apparent in incongruent trials preceded by incongruent trials (II), relative to incongruent preceded by congruent trials (CI) (averaged over safe and threat conditions) (a), but a positive affective bias is present only under safe conditions. (B), with enhanced processing of happy relative to fear faces only under safe conditions. $\mathrm{RT}=$ reaction time, $\mathrm{I}=$ incongruent, $\mathrm{C}=$ congruent, $\mathrm{F}=$ fearful face, $\mathrm{H}=$ happy face, $\mathrm{NS}=$ not significant; error bars represent standard errors of the means. ${ }^{*} p<.05$

Accuracy interaction with BAS reward seeking When BAS reward seeking was included as an extra factor in the accuracy ANOVA, modulation of positive bias by threat was shown to be influenced by individual differences in reward seeking [threat $\mathrm{x}$ valence $\mathrm{x}$ BAS interaction: $F(1$, $29)=7.1, p=.013]$. This effect was driven by a significant enhancement of fear-face accuracy under threat (.14 errors)

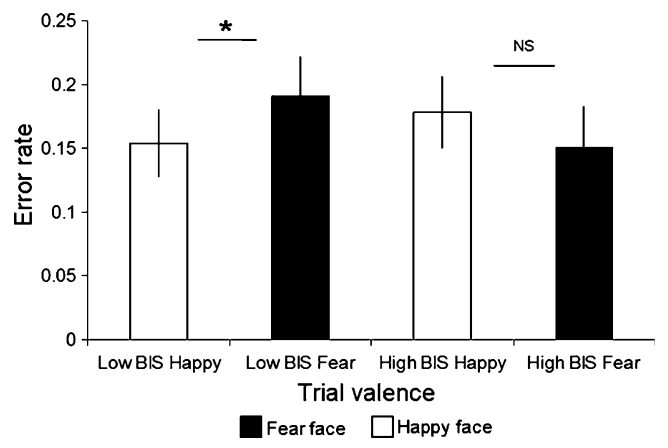

Fig. 3 A positive bias (increased accuracy for happy relative to fearful faces) was seen in low, but not in high, scorers on the BIS scale (averaged over conditions; error bars represent standard errors of the means, NS = nonsignificant). ${ }^{*} p<.05$ relative to safe (.20 errors) conditions in the low-BAS reward seeking group [main effect of condition on fear faces with low BAS: $F(1,29)=11.5, p=.002]$ but not in the high-BAS group [no effect of condition on fear faces with high BAS: $F(1,29)=3.4, p=.08]$. It should be noted that neither BIS nor BAS interaction was significant if it was included as a mean-centred continuous variable.

Correlation between accuracy and state measures The "happier" the participants rated themselves under the safe condition, the larger the difference in the number of errors was between conditions [see Fig. $4 ; r(28)=.57, p=.001$ ] . Moreover, the greater the difference in happy ratings across safe and fear conditions, the greater the enhancement in happy-face accuracy relative to fearful-face accuracy [i.e., the greater the positive bias; $r(30)=.28, p=.027]$. No state-measure scores correlated with BIS scores.

Face rating post-task

Subjects rated the fear and happy faces as equally salient $[F(1,27)=0.7$, n.s.]. As such, the valence effect on the Stroop task was not driven by a difference in salience between the happy and fearful faces.

\section{Discussion}

The present study examined the extent to which threat of shock, which is designed to mimic a key symptom of anxiety disorders, reproduced the alterations in cognitive performance that are characteristic of anxiety disorders. We showed that threat of shock induced affective biases similar to those seen in anxiety disorders, but did not, contrary to expectation, impair conflict adaptation. Threat of shock thus partially modelled the effects of generalized anxiety disorder and panic disorder on cognition by altering affective processing, whilst having no effect on cognitive

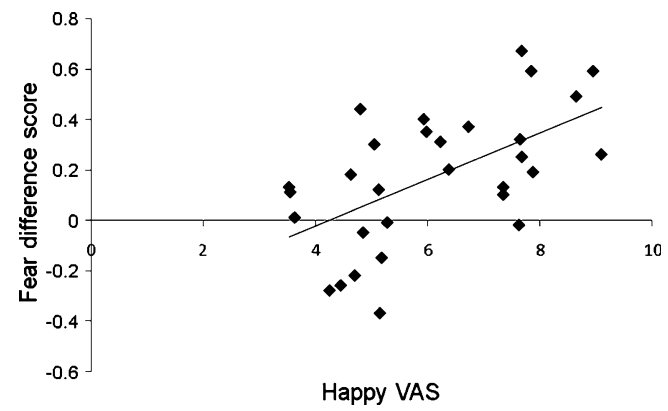

Fig. 4 The happier the individuals were, the more errors they made on fear trials under the safe condition relative to under the threat condition $[r(30)=.57, p=.001]$. VAS $=$ visual analogue scale 
rigidity. To the best of our knowledge, this is the first study to explicitly demonstrate affective bias in facial processing in response to threat of shock. A number of studies have shown that threat of shock elevates startle reflex (Grillon, 2008b; Lissek et al., 2007), reduces monetary reward anticipation (Ryan \& Diego, 2006), perturbs spatial working memory (Lavric et al., 2003; Shackman et al., 2006), and reduces sexual arousal (Beck, Barlow, Sakheim, \& Abrahamson, 1987), but the literature regarding the effect of threat of shock on affective biases is surprisingly sparse.

The effect of induced anxiety on affective biases

In healthy adults, threat of shock mimicked the effect of clinical anxiety on affective biases. Specifically, the threat of shock enhanced aversive affective processing and abolished a significant bias away from aversive processing (i.e., greater accuracy on happy relative to fearful faces, or a positive affective bias) in healthy individuals during the safe condition. From an adaptive perspective, such anxietyinduced enhancement of aversive processing could serve the purpose of increasing vigilance to danger in threatening environments (Grillon, 2008b). The specific direction of this bias, however, indicates a disproportionate inability to accurately process aversive stimuli in healthy individuals in safe conditions, which is then abolished by threat. From this perspective, the finding is reminiscent of the classic concept of depressive realism (Alloy \& Abramson, 1979), which states that healthy individuals can sometimes act in "blissful ignorance." It may be, for example, that inhibiting the processing of nonrelevant aversive processing (and, hence, making more errors in response to aversive information) under safe conditions is adaptive. Indeed, in a constantly changing and frequently nonthreatening environment, it would be cognitively wasteful to process all stressors. There is no adaptive purpose of worrying about one's inevitable death, for instance. As such, "blissful ignorance," or a relative deficit in accurately detecting fearful stimuli, may increase adaptive fitness under safe conditions (Cools, Roberts, \& Robbins, 2008) and may represent an endophenotype for resilience to anxiety disorders (Elliott, Sahakian, \& Charney, 2008). Such resilience could be driven by a combination of biological (e.g., oxytocin levels) and psychosocial (e.g., maternal behaviour and coping strategies; Altemus, 2006; Grillon, 2008a) factors that lead to elevated appetitive striatal processing of reward (Simon et al., 2009) and reduced amygdala-linked aversive processing (Cools et al., 2005) under safe conditions. Threatening conditions, however, reverse this situation, such that it becomes adaptive to focus on all aversive information in order to avoid harm. Thus, aversive processing is significantly enhanced under threat of shock.
The effect of induced anxiety on cognitive rigidity

Contrary to the effects of generalized anxiety disorder and panic disorder (Chechko et al., 2009; Etkin et al., 2010), however, threat of shock had no effect on cognitive rigidity, and it did not influence conflict adaptation. Moreover, the effect of threat on affective biases meanr that this lack of interaction cannot be attributed to a failure of the manipulation. This study is, to the best of our knowledge, the first to directly examine the effect of threat of shock on both affective biases and cognitive rigidity using a single paradigm and, as such, reveals a dissociation between the effects of experimentally induced anxiety on different facets of cognitive performance. This dissociation thus highlights a limitation of threat of shock as a model for anxiety disorders. It may be that the subjective state of anxiety, which is common to both threat and anxiety disorders, evokes the affective bias, but a more pervasive symptomatology, present in clinical anxiety but not in induced anxiety, is required to impair conflict adaptation. Indeed, anxiety disorders show complex alterations in neurotransmission (Deakin, 1998a, 1998b), neural activation (Etkin et al., 2006) and functional neuroconnectivity (Etkin \& Wager, 2007), any of which could impact conflict adaptation. In particular, conflict adaptation in the present task has been shown to rely on cortical regions including the rostral (pregenual) cingulate cortex, whereas processing of fearful faces involves subcortical regions such as the amygdala (Chechko et al., 2009; Egner et al., 2008; Etkin et al., 2006; Etkin et al., 2010; Etkin \& Wager, 2007). As such, a state of anxiety may influence (bottom-up) subcortical-related, but not (top-down) cortical-related, processing (Robinson \& Sahakian, 2009b). Ongoing research directly assessing the effects of threat of shock on functional neural activity will test this interpretation, but such dissociations highlight the need to treat all symptoms of anxiety disorders (i.e., affective symptoms as well as cognitive symptoms), since the relief of one symptom (e.g., the state of anxiety) may not accompany remission of others (e.g., cognitive rigidity; Elliott, Zahn, Deakin, \& Anderson, 2011). As highlighted in the introduction, much may be gained by developing experimental models of anxiety, but no single model will fully mimic anxiety disorders. It is therefore critical to define the overlaps and boundaries between our experimental models and the different anxiety disorders.

It should also be noted that a number of studies have demonstrated that individuals with high trait anxiety (McDermott et al., 2009; Righi, Mecacci, \& Viggiano, 2009; Sehlmeyer et al., 2010) or high state anxiety (Hajcak \& Foti, 2008; Righi et al., 2009; Tops \& Boksem, in press) show elevated neural indices of conflict monitoring as measured using electroencephalography (e.g., the ERN or 
N2). These findings suggest that neural processing of conflict, potentially in the dorsal anterior cingulate (Amodio et al., 2008), is actually elevated in individuals with increased anxiety. Indeed, two studies failed to demonstrate effects of threat (Shackman et al., 2011) and aversive stimuli (Wiswede et al., 2009) on conflict processing alongside such elevated electroencephalographic indices of conflict monitoring. Reconciling these findings with (1) our present failure to show an effect of threat of shock on conflict adaptation and (2) the previously reported abolished conflict adaptation in generalized anxiety disorders and panic disorder (Chechko et al., 2009; Etkin et al., 2010) presents an intriguing conundrum for future research.

\section{Individual differences}

Consistent with prior research (Krug \& Carter, 2010; Miu et al., 2008; Osinsky et al., 2010), anxiety-related cognitive processing was also modulated by individual differences. Low trait BIS scores predict low trait anxiety (Muris et al., 2001), reduced amygdala reactivity to fearful faces (Cools et al., 2005), reduced no-go N2 and ERN amplitudes (neural indices of conflict monitoring; Amodio et al., 2008), reduced impact of daily stressors on negative affect (Gable et al., 2000), and increased striatal response to reward (Simon et al., 2009). In the present study, individuals with low BIS scores showed a positive bias (i.e., made fewer errors on happy than on fearful face trials) that was not present in those with high BIS scores. Individual differences in BAS reward seeking also modulated the sensitivity of affective biases to threat of shock. Threat enhanced aversive processing in low-reward-seeking individuals only. A related but distinct effect was also seen in state "happiness" ratings, with happier individuals demonstrating a greater positive bias under safe relative to threat conditions. The present study was not optimized to assess these individual-difference effects, but the combination of trait measures with affective manipulations, such as threat of shock, provides a promising way to assess the link between trait anxiety and the impact of anxiogenic situations on cognitive and affective processing.

Putative mechanisms of threat-induced affective bias

The threat-mediated affective bias we have shown bears strong similarity to the effect of serotonin reduction (another anxiogenic manipulation; Grillon, Levenson, \& Pine, 2006) on aversive processing in healthy individuals; both manipulations remove a positive bias that is present in healthy individuals at baseline (Cools, Robinson, \& Sahakian, 2008). One explanation for the serotonergic bias effect is that cortically originating top-down serotenergic neurons inhibit aversive processing in, for example, the amygdala, the striatum, the bed nucleus of the stria terminalis, or the raphe nuclei (Cools, Roberts, \& Robbins, 2008; Robinson, Frank, Sahakian, \& Cools, 2010; Somerville, Whalen, \& Kelley, 2010). This inhibition generates a positive bias in information processing (Dayan \& Huys, 2008) that is removed (i.e., disinhibited) when serotonin is depleted. Such top-down disinhibitory mechanisms have also been proposed for the effect of anxiety on aversive processing (Etkin \& Wager, 2007; see also Eysenk's attentional control theory: Eysenck, Derakshan, Santos, \& Calvo, 2007). It is therefore plausible that the effect of threat of shock seen here may result from a similar top-down neural disinhibition of aversive processing. Such a mechanism would explain the elevated amygdala reactivity reported under threat of shock (Cornwell et al., 2007) and provide a plausible neural mechanism by which anxiety could increase vigilance to aversive stimuli. Speculating further, permanent disinhibition of such neurons could lead to the debilitating attentional bias towards aversive stimuli seen in anxiety disorders. However, whilst the findings can provide some insight into the mechanisms by which attentional biases are modulated by neurotransmitters, it is important to note that the present study did not include such manipulations, so caution is needed when making these inferences. Ongoing research examining the effect of serotonin reduction on conflict adaptation will, however, directly assess this hypothesis.

\section{Caveats and possible limitations}

It should be pointed out that the affective bias effect we saw is manifest as an alteration in accuracy, whereas the conflict adaptation effect is manifest as an alteration in RT. Thus, the affective bias we have shown here is more comparable to the affective biases seen in tasks such as reversal learning and go/no go tasks, where biases are revealed by accuracy effects (Clark, Chamberlain, \& Sahakian, 2009; Cools, Robinson, \& Sahakian, 2008; Robinson \& Sahakian, 2009b; Robinson, Standing, DeVito, Cools, \& Sahakian, 2010; Roiser et al., 2009), than to the affective biases seen on classic versions of the affective Stroop, in which biases are often revealed by RT differences (Ernst, 2010). The distinction between the conflict adaptation RT findings (which replicate prior findings: Egner et al., 2008; Etkin et al., 2010; Etkin \& Wager, 2007) and the affective accuracy findings may, however, reflect the nature of the processing impairment/enhancement driving each effect. Conflict adaptation may be dependent on top-down processes that impact RT, whilst affective biases may be driven by bottom-up processing that directly impacts accuracy. It should also be noted that the targets and distractors in the task used here (faces vs. words) are likely processed by distinct neural pathways. The task and stimuli have been well tested (Egner et al., 2008; Etkin et al., 2010; Etkin \& 
Wager, 2007; Frey et al., 2010; Krug \& Carter, 2010; Osinsky et al., 2010) and have several advantages over classic Stroop tasks (Buhle et al., 2010; Ernst, 2010), but care should be taken in comparing findings across tasks. Accordingly, the error rates in the present study are slightly higher than those reported in the original adapted task (Egner et al., 2008; however, not in all versions of the task: Frey et al., 2010). This discrepancy is likely related to the fact that in the present version of the task we recorded responses only during stimulus presentation, whereas in the task used by Egner et al., they also recorded slower poststimulus responses that occurred during the interstimulus interval (note that responses were also approximately $100 \mathrm{~ms}$ faster in the present task). Future research will adopt the exact same task utilised in prior work, but the unaffected conflict adaptation seen in the RT data across both conditions in the present study suggests that excluding these poststimulus responses did not bias the present data. Indeed, filtering out the slower responses would have, if anything, reduced the sensitivity of the task to detect conflict adaptation by trimming off the slowest (CI) responses and reducing the window of potential difference between II and CI responses. The lack of a conflict adaptation effect in accuracy suggests that equal numbers of all trial types were recorded.

As a final point, it should be noted that although in the secondary individual-difference analysis we adopted a frequently used method of dividing healthy subjects by individual differences (e.g., Krug \& Carter, 2010; Osinsky et al., 2010), this method was not optimal, and we may have trimmed off some of the most highly harm-avoidant individuals, who would not have agreed to participate in a threat-of-shock study.

\section{Conclusion}

Threat of shock in healthy individuals partially reproduces the altered cognitive performance seen in anxiety disorders. Specifically, threat of shock enhances aversive processing, potentially through a neural disinhibitory mechanism, but has no effect on conflict adaptation, which may be subserved by higher cortical regions uninfluenced by induced anxiety. Moreover, we revealed a positive bias under safe conditions, which may represent an endophenotype for resilience to emotional disorders (Elliott et al., 2008).

Financial disclosure The author(s) declare that, except for income received from the primary employer, no financial support or compensation has been received from any individual or corporate entity over the past 3 years for research or professional service, and there are no personal financial holdings that could be perceived as constituting a potential conflict of interest.

\section{References}

Alloy, L. B., \& Abramson, L. Y. (1979). Judgment of contingency in depressed and nondepressed students: Sadder but wiser? Journal of Experimental Psychology. General, 108, 441-485.

Altemus, M. (2006). Sex differences in depression and anxiety disorders: Potential biological determinants. Hormones and Behavior, 50, 534-538.

Amodio, D. M., Master, S. L., Yee, C. M., \& Taylor, S. E. (2008). Neurocognitive components of the behavioral inhibition and activation systems: Implications for theories of self-regulation. Psychophysiology, 45, 11-19.

Barrós-Loscertales, A., Meseguer, V., Sanjuán, A., Belloch, V., Parcet, M. A., Torrubia, R., et al. (2006). Behavioral inhibition system activity is associated with increased amygdala and hippocampal gray matter volume: A voxel-based morphometry study. Neuroimage, 33, 1011-1015.

Beck, J. G., Barlow, D. H., Sakheim, D. K., \& Abrahamson, D. J. (1987). Shock threat and sexual arousal: The role of selective attention, thought content, and affective states. Psychophysiology, 24, 165-172.

Beck, A. T., Ward, C. H., Mendelson, M., Mock, J., \& Erbaugh, J. (1961). An inventory for measuring depression. Archives of General Psychiatry, 4, 561-571.

Becker, E. S., Rinck, M., Margraf, J., \& Roth, W. T. (2001). The emotional Stroop effect in anxiety disorders: General emotionality or disorder specificity? Journal of Anxiety Disorders, 15, $147-159$.

Beddington, J., Cooper, C. L., Field, J., Goswami, U., Huppert, F. A., Jenkins, R., et al. (2008). The mental wealth of nations. Nature, $455,1057-1060$.

Bishop, S. J. (2007). Neurocognitive mechanisms of anxiety: An integrative account. Trends in Cognitive Sciences, 11, 307-316.

Buhle, J., Wager, T. D., \& Smith, E. E. (2010). Using the Stroop task to study emotion regulation. In R. R. Hassin, K. N. Ochsner, \& Y. Trope (Eds.), Self control in society, mind, and brain (pp. 93113). New York: Oxford University Press.

Carver, C. S., \& White, T. L. (1994). Behavioral inhibition, behavioral activation, and affective responses to impending reward and punishment: The BIS/BAS scales. Journal of Personality and Social Psychology, 67, 319-333.

Chechko, N., Wehrle, R., Erhardt, A., Holsboer, F., Czisch, M., \& Sämann, P. G. (2009). Unstable prefrontal response to emotional conflict and activation of lower limbic structures and brainstem in remitted panic disorder. PLoS ONE, 4, e5537.

Clark, L., Chamberlain, S. R., \& Sahakian, B. J. (2009). Neurocognitive mechanisms in depression: Implications for treatment. Annual Review of Neuroscience, 32, 57-74.

Cools, R., Calder, A., Lawrence, A., Clark, L., Bullmore, E., \& Robbins, T. (2005). Individual differences in threat sensitivity predict serotonergic modulation of amygdala response to fearful faces. Psychopharmacology, 180, 670-679.

Cools, R., Roberts, A. C., \& Robbins, T. W. (2008). Serotoninergic regulation of emotional and behavioural control processes. Trends in Cognitive Sciences, 12, 31-40.

Cools, R., Robinson, O. J., \& Sahakian, B. (2008). Acute tryptophan depletion in healthy volunteers enhances punishment prediction but does not affect reward prediction. Neuropsychopharmacology, 33, 2291-2299.

Cornwell, B. R., Baas, J. M. P., Johnson, L., Holroyd, T., Carver, F. W., Lissek, S., et al. (2007). Neural responses to auditory stimulus deviance under threat of electric shock revealed by spatially-filtered magnetoencephalography. Neuroimage, 37, 282-289.

Dayan, P., \& Huys, Q. J. M. (2008). Serotonin, inhibition, and negative mood. PLoS Computational Biology, 4, e4. 
Deakin, J. F. W. (1998a). Dysfunctional components of 5-HT neurotransmission in anxiety and depression. European Neuropsychopharmacology, 8, 58-59.

Deakin, J. F. W. (1998b). The role of serotonin in depression and anxiety. European Psychiatry, 13, 57s-63s.

Egner, T., Etkin, A., Gale, S., \& Hirsch, J. (2008). Dissociable neural systems resolve conflict from emotional versus nonemotional distracters. Cerebral Cortex, 18, 1475-1484.

Egner, T., \& Hirsch, J. (2005). Cognitive control mechanisms resolve conflict through cortical amplification of task-relevant information. Nature Neuroscience, 8, 1784-1790.

Ekman, P., \& Friesen, W. V. (1976). Pictures of facial affect. Palo Alto: Consulting Psychologists Press.

Elliott, R., Sahakian, B. J., \& Charney, D. S. (2008). State-of-science review: E7. The neural basis of resilience.U.K. Government's Foresight, Mental Capital and Wellbeing Project.

Elliott, R., Zahn, R., Deakin, J. F. W., \& Anderson, I. M. (2011). Affective cognition and its disruption in mood disorders. Neuropsychopharmacology, 36, 153-182.

Erk, S., Kleczar, A., \& Walter, H. (2007). Valence-specific regulation effects in a working memory task with emotional context. Neuroimage, 37, 623-632.

Ernst, M. (2010). Conflict adaptation in generalized anxiety disorder: Small paradigm twist, large scientific leap. The American Journal of Psychiatry, 167, 489-492.

Etkin, A., Egner, T., Peraza, D. M., Kandel, E. R., \& Hirsch, J. (2006). Resolving emotional conflict: A role for the rostral anterior cingulate cortex in modulating activity in the amygdala. Neuron, 51, 871-882.

Etkin, A., Prater, K. E., Hoeft, F., Menon, V., \& Schatzberg, A. F. (2010). Failure of anterior cingulate activation and connectivity with the amygdala during implicit regulation of emotional processing in generalized anxiety disorder. The American Journal of Psychiatry, 167, 545-554.

Etkin, A., \& Wager, T. D. (2007). Functional neuroimaging of anxiety: A meta-analysis of emotional processing in ptsd, social anxiety disorder, and specific phobia. The American Journal of Psychiatry, 164, 1476-1488.

Eysenck, M. W., Derakshan, N., Santos, R., \& Calvo, M. G. (2007). Anxiety and cognitive performance: Attentional control theory. Emotion, 7, 336-353.

First, M. B., Spitzer, R. L., Gibbon, M., \& Williams, J. B. W. (2002). Structured clinical interview for DSM-IV-TR: Axis I disordersPatient edition (SCID-I/P, 11/2002 Revision). New York: New York State Psychiatric Institute.

Frey, B. N., Hall, G. B., Attard, S., Yucel, K., Skelin, I., Steiner, M., et al. (2010). Shift in the brain network of emotional regulation in midlife women: Is the menopausal transition the turning point? Menopause, 17, 840-845. doi:10.1097/gme.0b013e3181df840f.

Gable, S. L., Reis, H. T., \& Elliot, A. J. (2000). Behavioral activation and inhibition in everyday life. Journal of Personality and Social Psychology, 78, 1135-1149.

Grillon, C. (2008a). Greater sustained anxiety but not phasic fear in women compared to men. Emotion, 8, 410-413.

Grillon, C. (2008b). Models and mechanisms of anxiety: Evidence from startle studies. Psychopharmacology, 199, 421-437.

Grillon, C., Levenson, J., \& Pine, D. S. (2006). A single dose of the selective serotonin reuptake inhibitor citalopram exacerbates anxiety in humans: A fear-potentiated startle study. Neuropsychopharmacology, 32, 225-231.

Hajcak, G., \& Foti, D. (2008). Errors are aversive. Psychological Science, 19, 103-108.

Hasler, G., van der Veen, J. W., Grillon, C., Drevets, W. C., \& Shen, J. (2010). Effect of acute psychological stress on prefrontal GABA concentration determined by proton mag- netic resonance spectroscopy. The American Journal of Psychiatry, 167, 1226-1231.

Hettema, J. M., Neale, M. C., \& Kendler, K. S. (2001). A review and meta-analysis of the genetic epidemiology of anxiety disorders. The American Journal of Psychiatry, 158, 1568-1578. doi:10.1176/appi.ajp.158.10.1568.

Kessler, R. C., Berglund, P., Demler, O., Jin, R., Merikangas, K. R., \& Walters, E. E. (2005). Lifetime prevalence and age-ofonset distributions of DSM-IV disorders in the national comorbidity survey replication. Archives of General Psychiatry, 62, 593-602.

Krug, M. K., \& Carter, C. S. (2010). Adding fear to conflict: A general purpose cognitive control network is modulated by trait anxiety. Cognitive, Affective \& Behavioral Neuroscience, 10, 357-371.

Kupfer, D. J., First, M. B., \& Regier, D. A. (2002). A research agenda for DSM- $V$. New York: American Psychiatric Association.

Lavric, A., Rippon, G., \& Gray, J. R. (2003). Threat-evoked anxiety disrupts spatial working memory performance: An attentional account. Cognitive Therapy Research, 27, 489-504.

Lissek, S., Orme, K., McDowell, D. J., Johnson, L. L., Luckenbaugh, D. A., Baas, J. M., et al. (2007). Emotion regulation and potentiated startle across affective picture and threat-of-shock paradigms. Biological Psychology, 76, 124-133.

McDermott, J. M., Perez-Edgar, K., Henderson, H. A., ChronisTuscano, A., Pine, D. S., \& Fox, N. A. (2009). A history of childhood behavioral inhibition and enhanced response monitoring in adolescence are linked to clinical anxiety. Biological Psychiatry, 65, 445-448.

McNaughton, N., \& Gray, J. A. (2000). Anxiolytic action on the behavioural inhibition system implies multiple types of arousal contribute to anxiety. Journal of Affective Disorders, 61, 161176.

Miu, A. C., Heilman, R. M., \& Houser, D. (2008). Anxiety impairs decision-making: Psychophysiological evidence from an Iowa Gambling Task. Biological Psychology, 77, 353-358.

Mogg, K., \& Bradley, B. (2005). Attentional bias in generalized anxiety disorder versus depressive disorder. Cognitive Therapy Research, 29, 29-45.

Muris, P., Merckelbach, H., Schmidt, H., Gadet, B., \& Bogie, N. (2001). Anxiety and depression as correlates of self-reported behavioural inhibition in normal adolescents. Behavior Research and Therapy, 39, 1051-1061.

Osinsky, R., Alexander, N., Gebhardt, H., \& Hennig, J. (2010). Trait anxiety and dynamic adjustments in conflict processing. Cognitive, Affective \& Behavioral Neuroscience, 10, 372-381.

Osman, A., Breitenstein, J. L., Barrios, F. X., Gutierrez, P. M., \& Kopper, B. A. (2002). The Fear of Pain Questionnaire III: Further reliability and validity with nonclinical samples. Journal of Behavioral Medicine, 25, 155-173.

Phelps, E. A., O'Connor, K. J., Gatenby, J. C., Gore, J. C., Grillon, C., \& Davis, M. (2001). Activation of the left amygdala to a cognitive representation of fear. Nature Neuroscience, 4, 437441.

Righi, S., Mecacci, L., \& Viggiano, M. P. (2009). Anxiety, cognitive self-evaluation and performance: ERP correlates. Journal of Anxiety Disorders, 23, 1132-1138.

Robinson, O. J., Frank, M. J., Sahakian, B. J., \& Cools, R. (2010). Dissociable responses to punishment in distinct striatal regions during reversal learning. Neuroimage, 51, 1459-1467.

Robinson, O. J., \& Sahakian, B. J. (2009a). Acute tryptophan depletion evokes negative mood in healthy females who have previously experienced concurrent negative mood and tryptophan depletion. Psychopharmacology, 205, 227-235. doi:10.1007/ s00213-009-1533-4. 
Robinson, O. J., \& Sahakian, B. J. (2009b). A double dissociation in the roles of serotonin and mood in healthy subjects. Biological Psychiatry, 65, 89-92.

Robinson, O., Standing, H., DeVito, E., Cools, R., \& Sahakian, B. (2010). Dopamine precursor depletion improves punishment prediction during reversal learning in healthy females but not males. Psychopharmacology, 211, 187-195.

Roiser, J. P., Levy, J., Fromm, S. J., Nugent, A. J., Talagala, S. L., Hasler, G., et al. (2009). The effects of tryptophan depletion on neural responses to emotional words in remitted depression. Biological Psychiatry, 66, 441-450.

Ryan, B., \& Diego, A. P. (2006). Acute stress reduces reward responsiveness: Implications for depression. Biological Psychiatry, 60, 1147-1154.

Sehlmeyer, C., Konrad, C., Zwitserlood, P., Arolt, V., Falkenstein, M., \& Beste, C. (2010). ERP indices for response inhibition are related to anxiety-related personality traits. Neuropsychologia, 48, 2488-2495.

Shackman, A. J., Maxwell, J. S., McMenamin, B. W., Greischar, L. L., \& Davidson, R. J. (2011). Stress potentiates early and attenuates late stages of visual processing. The Journal of Neuroscience, 31, $1156-1161$

Shackman, A. J., Sarinopoulos, I., Maxwell, J. S., Pizzagalli, D. A., Lavric, A., \& Davidson, R. J. (2006). Anxiety selectively disrupts visuospatial working memory. Emotion, 6, 40-61.
Simon, J. J., Walther, S., Fiebach, C. J., Friederich, H.-C., Stippich, C., Weisbrod, M., et al. (2009). Neural reward processing is modulated by approach- and avoidance-related personality traits. Neuroimage, 49, 1868-1874.

Somerville, L. H., Whalen, P. J., \& Kelley, W. M. (2010). Human bed nucleus of the stria terminalis indexes hypervigilant threat monitoring. Biological Psychiatry, 68, 416-424. doi:10.1016/j. biopsych.2010.04.002.

Spielberger, C. D. (1983). State-trait anxiety inventory. Palo Alto: Consulting Psychologists Press.

Tops, M., \& Boksem, M. A. S. (in press). Cortisol involvement in mechanisms of behavioral inhibition. Psychophysiology. doi:10.1111/j.1469-8986.2010.01131.x

Wiswede, D., Münte, T. F., Goschke, T., \& Rüsseler, J. (2009). Modulation of the error-related negativity by induction of shortterm negative affect. Neuropsychologia, 47, 83-90.

\section{Author Note}

This research was supported by the Intramural Research Program of the National Institute of Mental Health. We also acknowledge the anonymous reviewers, whose useful suggestions improved this article. 